\title{
Proximity Detection Prototype Adapted to a Work Environment
}

Gabriel Villarrubia, Alejandro Sánchez, Ignasi Barri, Edgar Rubión, Alicia Fernández, Carlos Rebate, José A. Cabo, Teresa Álamos, Jesús Sanz, Joaquín Seco, Carolina Zato, Javier Bajo, Sara Rodríguez, and Juan M. Corchado

\begin{abstract}
This article presents a proximity detection prototype that uses ZigBee technology. The prototype is primarily oriented to proximity detection within an office environment and some of the particular characteristics specific to such an environment, including the integration of people with disabilities into the workplace. This allows the system to define and manage the different profiles of people with disabilities, facilitating their job assimilation by automatically switching on or off the computer upon detecting the user's presence, or initiating a procedure that automatically adapts the computer to the personal needs of the user.
\end{abstract}

Keywords: Zigbee, proximity detection, wake on LAN, personalization.

\author{
Alejandro Sánchez · Javier Bajo \\ Universidad Pontificia de Salamanca, Salamanca, Spain \\ e-mail: \{asanchezyu, jbajope\} @usal.es \\ Gabriel Villarrubia · Carolina Zato · Sara Rodríguez · Juan M. Corchado \\ Departamento Informática y Automática, Universidad de Salamanca, Salamanca, Spain \\ e-mail: \{gvg, carol_zato, srg, corchado\} @usal.es \\ Ignasi Barri · Edgar Rubión · Alicia Fernández · Carlos Rebate \\ Indra, Spain \\ e-mail: \{ibarriv, erubion, afernandezde, crebate\}@indra.es \\ José A. Cabo - Teresa Álamos \\ Wellness Telecom, Spain \\ e-mail: $\{$ talamos, jacabo\} @wtelecom.es \\ Jesús Sanz · Joaquín Seco \\ CSA, Spain \\ e-mail: $\{$ jesus.sanz, joaquin. seco\}@esa.es
}

P. Novais et al. (Eds.): Ambient Intelligence - Software and Applications, AISC 153, pp. 51-58. springerlink.com

(C) Springer-Verlag Berlin Heidelberg 2012 


\section{Introduction}

Modern societies are characterized as much by technological advances as they are by social advances. With regards to technological advances, the rapid development of TIC has had a direct impact on our lifestyle, while the realm of social advances includes an increasing sensitivity to allow persons with disabilities, or those at risk of social exclusion, to lead a normal and independent life, which includes the very relevant ability to carry out and receive compensation for professional activity. The effective integration into the workplace of people with disabilities is an enormous challenge for society, and new technologies should provide solutions that can further this integration.

There are a number of obstacles that impede the ability of people with disabilities to integrate in the workforce, and the ability of businesses to include them in their staff. One of the biggest challenges of incorporating people with disabilities into the workforce is the question of autonomy and mobility.

Within the field of technologies specifically developed to facilitate the lives of people with disabilities, there have been many recent advances that have notably improved their ability to perform daily and work-related tasks, regardless of the type and severity of the disability. Nevertheless, the complete integration of these individuals in the society in general, and the workforce in particular, is still considered a challenge. Consequently, it is critical to provide new tools that can eliminate these barriers and facilitate the incorporation of this group of individuals into the workforce. This article presents a proximity detection prototype, specifically developed for a work environment, which can facilitate tasks such as activating and personalizing the work environment; these apparently simple tasks are in reality extremely complicated for some people with disabilities.

The rest of the paper is structured as follows: The next section introduces the technology used in the development of this prototype. Section 3 presents the most important characteristics of the prototype. Finally, section 4 explains some results and conclusions that were obtained.

\section{Technology Applied}

There are currently different types of sensors that can distinguish whether a person or object is located within its range of action. To begin, there are presence sensors whose purpose is to determine the presence of an object at a specified interval of distance. There are also proximity sensors, which can measure the distance from an initial point (usually the sensor itself) to the object within a range of action. Other types of sensors include ZigBee sensors, which have been successfully applied in schools, hospitals, homes, etc. ZigBee is a low cost, low power consumption, two-way wireless communication standard that was developed by the ZigBee Alliance [5]. It is based on the IEEE 802.15.4 protocol, and operates on the ISM (Industrial, Scientific and Medical) band at $868 / 915 \mathrm{MHz}$ and a $2.4 \mathrm{GHz}$ spectrum. Due to this frequency of operation among devices, it is possible to transfer materials used in residential or office buildings while only minimally 
affecting system performance [1]. Although this system can operate at the same frequency as Wi-Fi devices, the possibility that it will be affected by their presence is practically null, even in very noise environments (electromagnetic interference). ZigBee is designed to be embedded in consumer electronics, home and building automation, industrial controls, PC peripherals, medical sensor applications, toys and games, and is intended for home, building and industrial automation purposes, addressing the needs of monitoring, control and sensory network applications [5]. ZigBee allows star, tree or mesh topologies. Devices can be configured to act as network coordinator (control all devices), router/repeater (send/receive/resend data to/from coordinator or end devices), and end device (send/receive data to/from coordinator) [6].

The IEEE 802.15.4 standard is designed to work with low rate networks with limited resources [2]. Furthermore, ZigBee incorporates an additional network with security and application layers under the IEEE 802.15.4 standard, and allows more than 65,000 connected nodes.

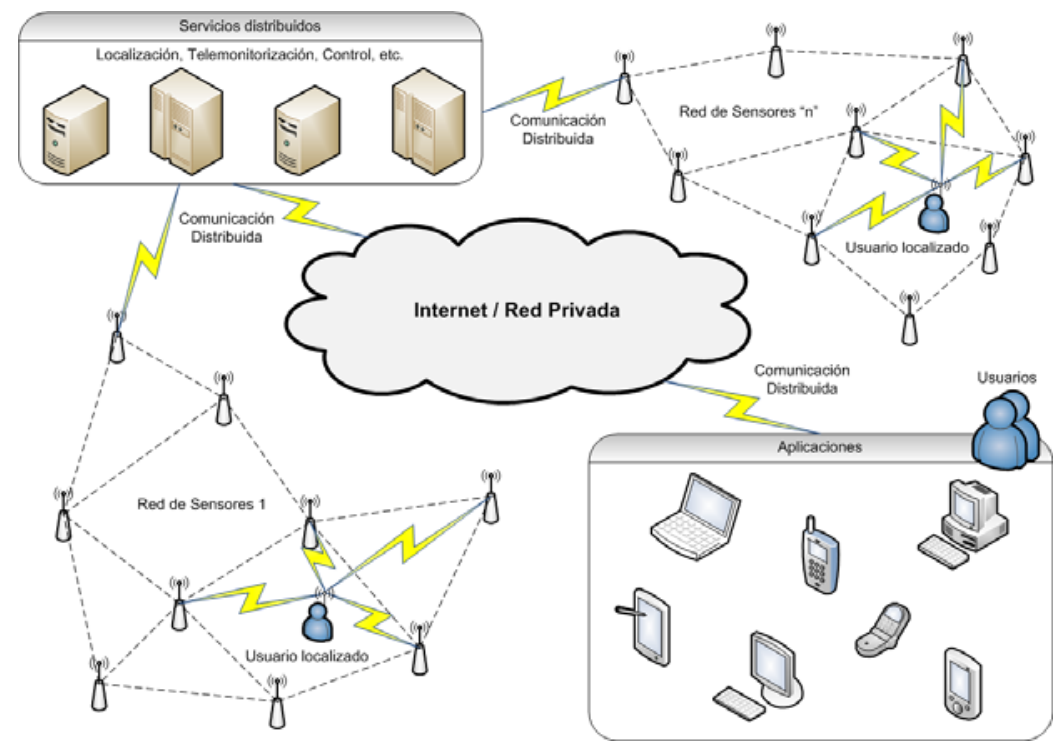

Fig. 1 Diagram of the Zigbee network

One of the main advantages of this system is that, as opposed to GPS type systems, it is capable of functioning both inside and out with the same infrastructure, which can quickly and easily adapt to practically any applied environment. The infrastructure of the proposed system is dynamic, adaptable, scalable, non-intrusive, and has a low operation cost. 


\section{Prototype}

\subsection{Architecture}

This document presents a proximity detection system that will be used by people with disabilities to facilitate their integration in the workplace. The main goal of the system is to detect the proximity of a person to a computer using ZigBee technology. This allows an individual to be identified, and for different actions to be performed on the computer, thus facilitating workplace integration: automatic switch on/off of the computer, identifying user profile, launching applications, and adapting the job to the specific needs of the user.

Every user in the proposed system carries a Zigbee tag, while a ZigBee reader is located in each system terminal. Thus, when a user tag is sufficiently close to a specific terminal, the reader can detect the user tag and immediately send a message to the ZigBee network coordinator.

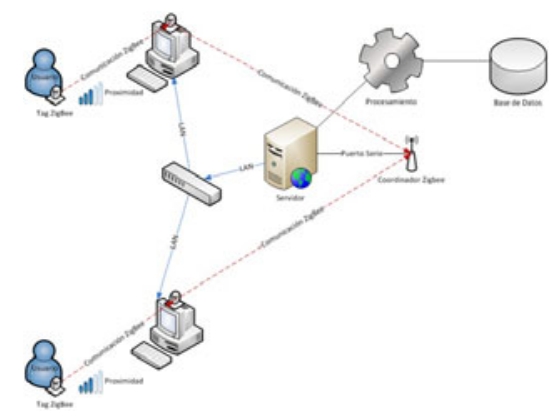

Fig. 2 System architecture

Figure 2 displays the basic operation of the proposed system. The components used in the system are:

Server: The primary system agents are deployed on this device: the manager agent, which manages communication and events; and the profile manager agent, which is responsible for managing user profiles. The agent functions were originally implemented with a traditional server.

Clients: These are user agents located in the client computer and are responsible for detecting the user with ZigBee technology, and for sending the user's identification to the manager agent. These agents are responsible for requesting the profile role adapted for the user.

Data base: The system uses a data base, which stores data related to the users, sensors, computer equipment and status, and user profiles.

ZigBee Coordinator: A ZigBee device responsible for coordinating the other ZigBee devices in the office. It is connected to the server by a serial port, and receives signals from each of the ZigBee tags in the system.

ZigBee Reader: These are ZigBee devices that are used to detect the presence of a user. Each ZigBee reader is located in a piece of office equipment (computer). 
ZigBee Tag: These are ZigBee devices carried by the user. When a tag is located near a reader (within a range defined according to the strength of the signal), it initiates the process of detecting proximity.

Network infrastructure: The system uses a LAN infrastructure that uses the wake-on-LAN protocol for the remote switching on and off of equipment.

\subsection{Wake On Lan}

Wake-on-LAN/WAN is a technology that allows a computer to be turned on remotely by a software call. It can be implemented in both local area networks (LAN) and wide area networks (WAN) [4]. It has many uses, including turning on a Web/FTP server, remotely accessing files stored on a machine, telecommuting, and in this case, turning on a computer even when the user's computer is turned off [7].

The Wake On LAN protocol defines a package called "Magic Package". This package contains the MAC address of the machine that it is desired to switch on. The Magic Package is sent by the link data layer to all the NICs (Network Interface Controller) using the address of the network diffusion. The frame is formed by 6 bytes (FF FF FF FF FF FF) followed of 16 repetitions of 48 bits that represents the MAC address. The frame is 102 bytes in total.

\begin{tabular}{|c|c|c|c|}
\hline FF-FF-FF-FF-FF-FF & Ethernet Mac Address & Ethernet Mac Address & Ethernet Mac Address \\
\hline Ethernet Mac Address & Ethernet Mac Address & Ethernet Mac Address & Ethernet Mac Address \\
\hline Ethernet Mac Address & Ethernet Mac Address & Ethernet Mac Address & Ethernet Mac Address \\
\hline Ethernet Mac Address & Ethernet Mac Address & Ethernet Mac Address & Ethernet Mac Address \\
\hline Ethernet Mac Address & \multicolumn{3}{|c|}{6 Bytes } \\
\cline { 2 - 3 }
\end{tabular}

Fig. 3 Wake-on-LAN magic packet

\subsection{Detection System}

The proposed proximity detection system is based on the detection of presence by a localized sensor called the control point, which has a permanent and known location. Once the object has been detected and identified, its location is delimited within the proximity of the sensor that identified it. Consequently, the location is based on criteria of presence and proximity, according to the precision of the system and the number of control points displayed.

The parameter used to carry out the detection of proximity is the RSSI (Received Signal Strength Indication), a parameter that indicates the strength of the received signal. This force is normally indicated in $\mathrm{mW}$ or using logarithmic units $(\mathrm{dBm}) .0 \mathrm{dBm}$ is equivalent to $1 \mathrm{~mW}$. Positive values indicate a signal strength greater than $1 \mathrm{~mW}$, while negative values indicate a signal strength less than $1 \mathrm{~mW}$.

Under normal conditions, the distance between transmitter and receiver is inversely proportional to the RRSI value measured in the receiver; in other words, 
the greater the distance, the lower the signal strength received. This is the most commonly used parameter among RTLS.

As shown in Figure 3, the tag located between two readers will be recognized by each reader with different RSSL levels.

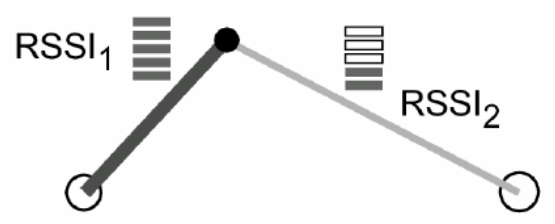

Fig. 4 Diagram of proximity distance detection based on RSSI

RSSI levels provide an appropriate parameter for allowing our system to function properly. However, variations in both the signal transmission and the environment require us to define an efficient algorithm that will allow us to carry out our proposal. This algorithm is based on the use of a steps or measurement levels ( 5 levels were used), so that when the user enters the range or proximity indicated by a RSSI level of -50 , the levels are activated. While the values received are less than the given range, each measurement of the system activates a level. However, if the values received fall outside the range, the level is deactivated. When the maximum number of levels has been activated, the system interprets this to mean that the user is within the proximity distance of detection and wants to use the computer equipment. Consequently, the mechanisms are activated to remotely switch on both the computer and the profile specific to the user's disability.

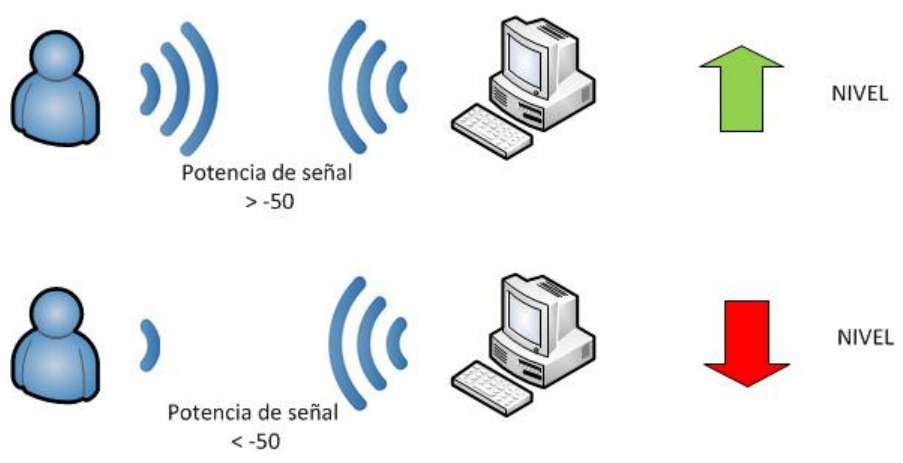

Fig. 5 System operation according to levels

The system is composed of 5 levels. The tags default to level 0 . When a user moves close to a marker, the level increases by one unit. The perceptible zone in the range of proximity gives an approximate RSSI value of -50 . If the user moves away from the proximity area, the RSSI value is less than -50 , resulting in a 
reduction in the level. When a greater level if reached, it is possible to conclude that the user has remained close to the marker, and the computer will be turned on.

On the other hand, reaching an initial level of 0 means that the user has moved a significant distance away from the workspace, and the computer is turned off.

\section{Results and Conclusions}

This document has presented a proximity detection prototype based on the use of $\mathrm{ZigBee}$ technology. The prototype is notably superior to existing technologies using Bluetooth, infrareds or radiofrequencies, and is highly efficient with regards to detection and distance. Additionally, different types of situations in a work environment were taken into account, including nearby computers, shared computers, etc.

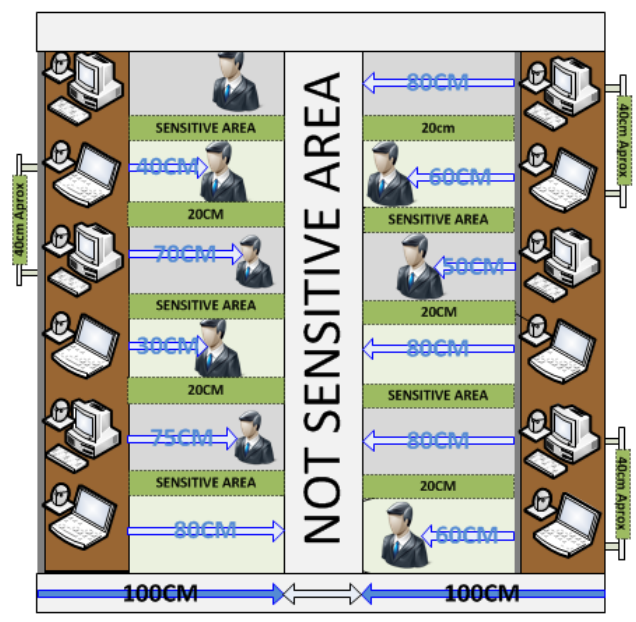

Fig. 6 Diagram of a work environment

Figure 6 represents a possible distribution of computers and laptops in a real office environment, separated by a distance of 2 meters. The activation zone is approximately $90 \mathrm{~cm}$, a distance considered close enough to be able to initiate the activation process. It should be noted that there is a "Sensitive Area" in which it is unknown exactly which computer should be switched on; this is because two computers in close proximity may impede the system's efficiency from switching on the desired computer. Tests demonstrate that the optimal distance separating two computers should be at least $40 \mathrm{~cm}$.

The prototype is specifically oriented to facilitate the integration of people with disabilities into the workplace. The detection and identification of a user makes it possible to detect any special needs, and for the computer to be automatically adapted for its use. The video in [3] confirms the operation of the prototype. 
Acknowledgements. This project has been supported by the Spanish CDTI. Proyecto de Cooperación Interempresas. IDI-20110343, IDI-20110344, IDI-20110345. Project supported by FEDER funds.

\section{References}

1. Huang, Y., Pang, A.: A Comprehensive Study of Low-power Operation in IEEE 802.15.4. In: Preceeding of the 10th ACM Symposium on Modeling, Analysis and Simulation of Wireless and Mobile Systems, Chaina, Crete Island, Greece (2007)

2. Singh, C.K., et al.: Performance evaluation of an IEEE 802.15.4 Sensor Network with a Star Topology (2008)

3. Universidad Pontificia de Salamanca. (En línea) (2011),

http: / /www . youtube. com/watch?v=9iYX-xney6E

4. Lieberman, P.: Wake on LAN Technology, White paper (2011), http: / /www. liebsoft.com/pdfs/Wake_On_LAN.pdf

5. ZigBee Standards Organization: ZigBee Specification Document 053474r13. ZigBee Alliance (2006)

6. Tapia, D.I., De Paz, Y., Bajo, J.: Ambient Intelligence Based Architecture for Automated Dynamic Environments. In: Borrajo, D., Castillo, L., Corchado, J.M. (eds.) CAEPIA 2007, vol. 2, pp. 151-180 (2011)

7. Nedevschi, S., Chandrashekar, J., Liu, J., Nordman, B., Ratnasamy, S., Taft, N.: Skilled in the art of being idle: reducing energy waste in networked systems. In: Proceedings of the 6th USENIX Symposium on Networked Systems Design and Implementation, Boston, Massachusetts, April 22-24, pp. 381-394 (2009) 